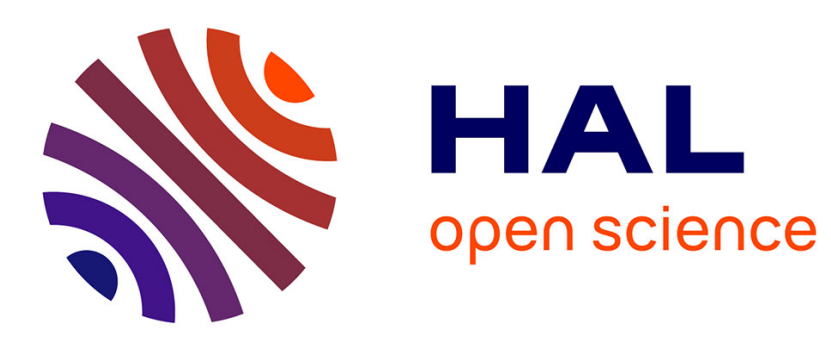

\title{
Homotopy Route Generation Model for Robust Trajectory Planning
}

Andrija Vidosavljevic, Daniel Delahaye, Vojin Tošić

\section{To cite this version:}

Andrija Vidosavljevic, Daniel Delahaye, Vojin Tošić. Homotopy Route Generation Model for Robust Trajectory Planning. ENRI. Air Traffic Management and Systems II, 420, Springer, pp 69-88, 2017, Lecture Notes in Electrical Engineering, 978-4-431-56421-8. 10.1007/978-4-431-56423-2_4 . hal01234103

\section{HAL Id: hal-01234103 \\ https://hal-enac.archives-ouvertes.fr/hal-01234103}

Submitted on 26 Nov 2015

HAL is a multi-disciplinary open access archive for the deposit and dissemination of scientific research documents, whether they are published or not. The documents may come from teaching and research institutions in France or abroad, or from public or private research centers.
L'archive ouverte pluridisciplinaire HAL, est destinée au dépôt et à la diffusion de documents scientifiques de niveau recherche, publiés ou non, émanant des établissements d'enseignement et de recherche français ou étrangers, des laboratoires publics ou privés. 


\title{
[EN-A-032] Homotopy Route Generation Model for Robust Trajectory Planning
}

\author{
${ }^{+}$A. Vidosavljevic*, D. Delahaye*, V. Tosic** \\ *MAIAA-Applied Mathematics Laboratory \\ Ecole Nationale de l'Aviation Civile (ENAC) \\ Toulouse, France \\ [vidosavljevic|delahaye]@ recherche.enac.fr \\ ** Division of Airports and Air Traffic Safety \\ Faculty of Transport and Traffic Engineering \\ University of Belgrade \\ Belgrade, Serbia \\ v.tosic@sf.bg.ac.rs
}

\begin{abstract}
Although advance future avionics will enable full compliance with the given trajectory; there are many uncertainty sources that can deflect aircraft from their intended positions. In this article we investigate potential of robust trajectory planning, considered as an additional demand management action, as a mean to alleviate the en-route congestion in airspace. Robust trajectory planning (RTP) involves generation of congestion-free trajectories with minimum operating cost taking into account uncertainty of trajectory prediction and unforeseen event. The model decision variables include ground delay, change of horizontal route and vertical profile (flight level) to resolve congestion problem. The article introduces a novel approach for route generation (3D trajectory) based on homotopic feature of continuous functions. It is shown that this approach is capable of generating a large number of route shapes with a reasonable number of decision variables. RTP problem is modeled as a mixed-variable optimization problem and it is solved using stochastic optimization technique. The model is tested on a real-life example from the French airspace. The results indicate that, under certain conditions, with small increase of total planned costs, it is possible to increase robustness of the proposed solution providing a good alternative to the solutions given by existing conflict-free trajectory planning models.
\end{abstract}

Keywords: 4D trajectory management, robust trajectory planning, Homotopy route generation model

\section{INTRODUCTION}

Even at the current level of traffic demand, en-route congestion is cited as one of the principals restricting factor to future growth of the airline industry. In 2012 , about $17 \%$ of (or 1.5 million) flights in Europe arrived with more than 15 minutes delay compared with the schedule [1]. For the same year, IATA [2] has estimated that delays increased airline direct operating cost by 4.5 billion Euros. With global air traffic demand expected to continue growing (predicted to be tripled by 2050 [3]), ICAO foreseen fundamental change in the operating paradigm for air navigation services in the following decade [4]. Major systems development programs are underway around the world, including the Next Generation Air Transportation System (NextGen) program in the United States [5], and the Single European Sky ATM Research (SESAR) program in Europe [6].

Trajectory Based Operations (TBO) represents a cornerstone of future ATM. TBO will enable aircraft to fly negotiated flight paths, Reference Business Trajectory (RBT), taking into consideration both operator preferences (more direct and fuel efficient routes) and optimal airspace system performance. Due to the high precision of RBT, TBO implies the possibility to design efficient congestion-free aircraft trajectories more in advance (pre-tactical, strategic level). Still, there are many uncertainty sources that may deflect an aircraft from its intended position (initial delay, wind, atmospheric temperature, actual aircraft mass, etc.) in addition to special events such as severe weather, volcanic ash, ATC strikes, etc. The best planning algorithm, however, is useless if the resulting plans cannot be implemented in the real world.

Therefore, in this work, model for robust 4D trajectory planning is proposed, taking into account uncertainty of trajectory prediction and unforeseen event, while minimizing total additional cost incur to the airspace users due to deviation from the user preferred trajectories (UPT).

This paper is organized as follows. Section 2 contains a brief overview of the existing methods for trajectory planning. In the next section 3 , a new method for robust trajectory planning (RTP) is proposed including Homotopy route generation model. The proposed RTP model is applied on a large scale real-life 
problem and test results are shown in section 4. Finally, section 5 sums up the findings and lists the major contributions of this work and point toward areas of future research.

\section{PREVIOUS RESEARCH}

In recent years, problem of trajectory planning becomes increasingly popular. Several classes of methods are used to address this problem. One of the earliest approaches [7] is based on the Evolution theory and uses basic operators: selection, mutation and crossover for generating new population of aircraft trajectories. The state space is a set of finite horizontal (straight line, turning point and offset) and vertical (level-off) maneuvers, similar to the ones used currently by ATCo. Their future work [8] and [9] took into account speed uncertainty and real aircraft performance model, in order to get closer to real ATC system. In more recent work [10], the authors propose a new framework that separates the trajectory prediction and detection model from the solver. Hence, for a given scenario a 4D-matrix, containing conflict information indexed by aircraft pairs and maneuver pairs, is computed using any simulator. This $4 \mathrm{D}$-matrix provides all the necessary data for the solver and enables the use and comparison of various algorithms on the same problem instances. Another approach for conflict resolution is shown in [11], where a sequence of maneuvers is controlled by the Particle Swarm Optimization (PSO) algorithm. Although these models generate feasible trajectories from aircraft performance perspective and take maneuvers cost into account, unfortunately they are not adapted with curved trajectories.

Another class of methods, based on a force field, enables automatic generation of conflict-free trajectories with a mathematical proof. Beside difficulties imposed by aircraft performance (speed limits or trajectory smoothness) [12], there are successful implementations of the force field methods, like [13], [14] and [15]. Even though, the major drawbacks of a force field method are a continuous aircraft maneuver in response to the changing force field [16], and complete neglect of the optimization.

In [12] new methodology for trajectory planning, using B-spline, is presented. Tactical aircraft conflict resolution is formulated as an optimization problem whose decision variables are the spline control points. In this work aircrafts were represented using a kinematic model to solve conflicts on the level flight (2D), evidencing local aspect of the method. Uncertainty of trajectory prediction is not considered as well. Another trajectory dimension reduction technique using linear piecewise interpolation is presented in [17] and [18]. In this work strategic de-confliction of aircraft trajectories is addressed with the objective to minimize the number of conflicts using route and slot allocation. Uncertainty in aircraft position is taken through "freedom margin" that is slightly higher than the separation norm (6 NM), while the cost of the solution is not considered. Trajectory design based on the wavefront propagation principle termed, light propagation algorithm (LPA), is shown in [19]. Propagation is discretized in space and time and a branch-and-bound algorithm is used to compute smooth geodesic with static and dynamic obstacles. In order to deal with the conflict resolution problem, LPA controls sequentially aircraft trajectories by selecting aircraft according to some given priority rule. This lead to unfairness between aircrafts particularly for problem instances involving many aircrafts, limiting application of LPA. The limitation is partly overcome using shifting time window [20] that, however, doesn't guarantee global optimum. LPA extension, that includes longitudinal uncertainty of aircraft position, is presented in [20]. Even with the use of uncertainty reduction techniques, like Required Time of Arrival, there were still some remaining conflicts (around 10\%).

The most of existing trajectory planning models consider finding of conflict-free trajectories barely taking into account uncertainty of trajectory prediction. It is shown in this work that in the case of traffic disturbances, it is better to have a robust solution otherwise newly generated congestion problems would be hard and costly to solve.

\section{ROBUST TRAJECTORY PLANNING}

\subsection{Problem statement}

In this work we present an alternative way dealing with uncertainty in aircraft position (inability to cope with RBT) and unplanned situation, through building a more robust flight plan at a pre-tactical or strategic level. Robustness includes both reducing the likelihood of disruption and increasing the number of options to recover from a disruption easily. This way, flight plan itself becomes less affected by such disturbances reducing the need for tactical actions. Further, it positively affects the tactical controller workload alleviating traffic management and conflict resolution tasks, which is the primary target of future ATM [4].

Therefore method proposed in this work aims at generating set of robust 4D trajectories while minimizing total additional cost incur to the airspace users due to deviation from the UPTs. Although adding robustness to flight plan could result in higher airline planned costs, disruption costs (cost of management actions taken to resolve conflict on tactical level due to disruptions) might be reduced due to reduced need for tactical interventions, hopefully leading to reduction in airline real operating costs.

RTP problem is formulated as a multi-objective problem that addresses how to optimally generate a set of 4D trajectories from origin to destination, based on assignment of: horizontal route shape (2D route), 
vertical profile and slot of departure, in order to manage the two confronted objectives: maximizing total robustness, and minimizing total planned operating costs. It aims at finding system optimum (SO) solution, a solution that is optimal from a viewpoint of a system as a whole. RTP might be further constrained by ATS capacities (airport capacity), or may include no-fly zones (severe weather cells, restricted or prohibited zones, etc.) that flights should avoid.

\subsection{Robustness and flight interaction}

To increase robustness, one must first define what robustness is, as definition is usually problem dependent [21]. ATM is a safety critical system, with the main task to provide a safe flow of air traffic before making it punctual and expeditious, therefore in this work, robustness is considered as "the ability of a system to resist changes without adapting its initial stable configuration" [22].

Another question concerns: disturbances that have to be taken into account, and indicators that quantify the robustness [23]. There are many uncertainty sources that can deflect an aircraft from its intended position (initial delay, wind, atmospheric temperature, actual aircraft weight, etc.). Difference between aircraft actual position and planned position may happen in space and/or time. Taking into account advanced future avionics [24], the trajectory will conform to the flight plan in the spatial dimension, while the position on trajectory may be subject to deviations in the temporal dimension [25]. Time uncertainty of position is, thus, main disturbance considered in this work.

Various indicators may be used to characterize the robustness in the context of trajectory planning. An alternative way of quantifying the solution robustness is to measure its vulnerability to environmental change as opposite indicator. While the robustness describes the strength of a solution, the vulnerability describes its weakness [23]. In this work, flight interaction is chosen as a measure of solution vulnerability and indirect measure of solution robustness.

Interaction between two flights is defined as a situation where flights compete for the same point in 4D space at the planning level. Unlike a conflict that has a fixed separation norm [26]; interaction takes into account the aircraft position uncertainty propagation (deviations from the RBT). When aircraft positions coincide in $4 \mathrm{D}$ space it results in maximum interaction that decreases while the distance (in space and time) between them increases. In the presence of uncertainty, minimization of interaction between flights at planning level decreases conflict probability and thus increases solution robustness.

In this work, flight interaction is defined as an exponential function of time separation, i.e. difference between times of arrival at conflicting points. For a pair of conflicting points $p_{i}$ and $p_{j}$, with time separation $T S_{p_{i} p_{j}}$, interaction magnitude is computed by (1):

$$
I_{p_{i} p_{j}}=\left\{\begin{array}{cl}
e^{-\tau \cdot T s_{p_{i} p_{j}}}, & T S_{p_{i} p_{j}}<T S^{*} \\
0 & T S_{p_{i} p_{j}} \geq T S^{*}
\end{array}\right.
$$

where parameter $\tau$ controls the steepness of the exponential function and $T S^{*}$ bounds flight interaction. Both parameters might vary depending on the test scenario.

Conflicting points are a pair of route positions that are separated less than given 3D norm. Usually those are points of routes intersection; however, they could be closely separated points of non-intersecting routes. With classical trajectory representation, as an ordered list of position vectors (samples) in $4 \mathrm{D}$, brute force approach for detection of conflicting points implies a pairwise comparison of trajectory samples. This is, however, a time consuming process and not suitable for a problem that involves a large number of samples [27]. In this work, a grid-based scheme developed in [28] is used for position vector comparison. In this approach, airspace is discretized using a four dimension grid with each cell having a unique address. Every position vector is associated with the address of the cell in the grid where it belongs, and its interaction against all other vectors is computed comparing only vectors belonging to that and surrounding cells.

With curved flight routes multiple conflicting points might exist. All those points are taken into account, because the number of conflicting points quantifies interaction between two flights. Finally, interaction between two flights $f_{i}$ and $f_{j}$ is computed taking into account both magnitude of interaction between conflicting points and their quantity as in (2).

$$
I_{f_{i} f_{j}}=\sum_{\left\{\left(p_{i}, p_{j}\right) \mid p_{i} \in f_{i} \wedge p_{j} \in f_{j} \wedge \overline{p_{l} p_{J}} \leq D\right\}} I_{p_{i} p_{j}}
$$

where $D$ represents conflicting points separation norm.

\subsection{Route generation model}

Both, NextGen and SESAR perceive more direct, fuel efficient routes in the future ATM enabled by satellite positioning and advance navigation technologies. This will free airspace from the "old highways in the sky" [5]. Although there is only one shortest direct route (geodesic) for two points, as it may be unavailable due to obstacles or traffic congestion, or it might not be optimal taking into account wind on the route, alternative routes has to be considered. With curve-shape trajectories infinitive number of alternatives exist. Consequently, the set of possible curve routes is infinitely large. Curves are objects belonging to spaces with infinite dimensions, and in order to manipulate such objects it is necessary to reduce the dimension of the search space [29].

In this work homotopy is used as a dimension reduction technique. It has been observed that homotopic feature of continuous functions may be used 
to easily map large continuous spaces using only a small number of parameters. This feature is exploited in the route generation model to design the shape of the horizontal route (alternative horizontal route) that is matched with vertical profile to produce 3D route. Finally, 3D shapes are completed with time dimension in order to create trajectories.

\subsubsection{Homotopy}

In topology, two continuous functions are called homotopic if one can be "continuously deformed" into the other. Such a deformation is called a homotopy between the two functions, and the concept was first formulated by Poincaré around 1900 [30]. Formally, a homotopy between two continuous functions $f$ and $g$ from a topological space $X$ to a topological space $Y$ is defined to be a continuous function $H: X \times[0,1] \mapsto Y$ such that $H(x, 0)=f(x)$ and $H(x, 1)=g(x)$ for $\forall x \in X[31]$.

Dashed lines in Fig. 1 represent iso-contours of the homotopy $H(t, \alpha)=\alpha \cdot \gamma_{0}(t)+(1-\alpha) \cdot \gamma_{1}(t)$ in $\mathbb{R}^{2}$ computed as a convex combination of the reference functions $\gamma_{0}(t)$ and $\gamma_{1}(t)$ for different values of the parameter $\alpha$.

The route generation model uses homotopy $H(t, \alpha)$ to map the space between reference functions using single real number $\alpha$. Consequently, the model performance (resulting routes) is only influenced by the selection of the reference functions. Selection is further constrained as the route geometry has critical influence on the feasibility and performance of aircraft route tracking [14]. The list of reference function properties and selection process are presented in [32].

\subsubsection{Mathematical formulation}

Fig. 2 shows a pair of symmetric reference functions $h(x)$ and $-h(x)$. For a given $x$, two points on reference functions are represented as vectors in Cartesian coordinate system by $\mathbf{p}_{1}=(x, h)$ and $\mathbf{p}_{2}=(x,-h)$, where $h=h(x)$. Hence a point $\mathbf{p}$ of homotopy, defined as a convex combination with respect to symmetric reference functions, is given by (3).

$$
\begin{aligned}
& \mathbf{p}=\alpha \cdot \mathbf{p}_{1}+(1-\alpha) \cdot \mathbf{p}_{2} \\
& x_{p}=\alpha \cdot x+(1-\alpha) \cdot x=x \\
& y_{p}=\alpha \cdot h+(1-\alpha) \cdot(-h)=(2 \alpha-1) h \\
& \mathbf{p}=\mathbf{p}(x, \alpha)=(x,(2 \alpha-1) h(x))
\end{aligned}
$$

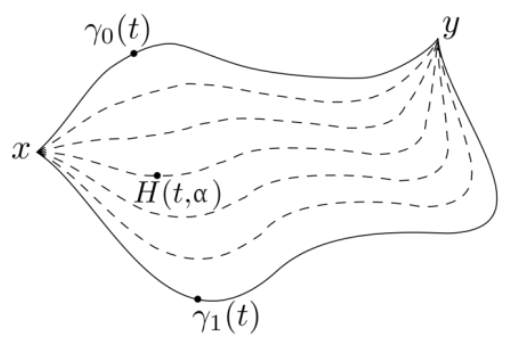

Figure 1: Example of two homotopic curves in $\mathbb{R}^{2}$

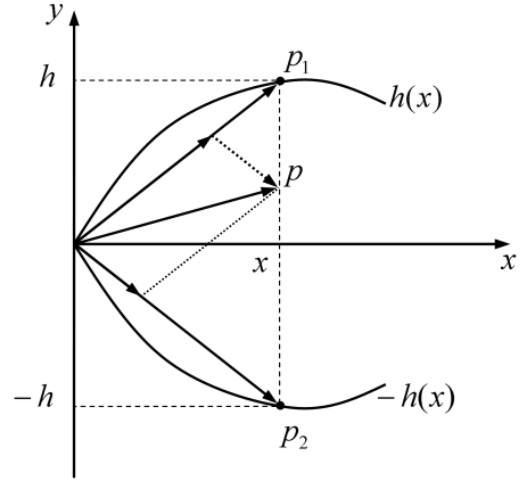

Figure 2: Symmetric homotopy with respect to ref. function $h(x)$

In the same manner, homotopies with respect to symmetric reference functions $h^{1}(x), h^{2}(x)$, etc. are given by $\mathbf{p}^{\mathbf{1}}\left(x, \alpha^{1}\right), \mathbf{p}^{2}\left(x, \alpha^{2}\right)$, etc. respectively. Finally, multiple homotopy, based on those reference functions, is computed by (4), as a weighted arithmetic mean of corresponding homotopies. Weights $\left|\alpha-\frac{1}{2}\right|$ represent an absolute deviation of parameter $\alpha$ from $\frac{1}{2}$, that is considered 'identity element' because the resulting symmetric homotopy is direct (nominal) route.

$$
\begin{aligned}
& \mathbf{p}=\mathbf{p}\left(x, \alpha^{1}, \alpha^{2}, \ldots\right)=\left(x, \sum_{i} Q^{i} \cdot h^{i}(x)\right) \\
& Q^{i}=Q^{i}\left(\alpha^{1}, \alpha^{2}, \ldots\right)=\frac{2\left|\alpha^{i}-\frac{1}{2}\right| \cdot\left(\alpha^{i}-\frac{1}{2}\right)}{\sum_{j}\left|\alpha^{j}-\frac{1}{2}\right|}
\end{aligned}
$$

\subsubsection{Alternative route design}

Due to physical constraints on airports, it is assumed, in this research, that area around airports and TMA will remain controlled. Therefore the horizontal route shape is only modified in the en-route segment. The route generation process is summarized in Fig. 3 .

In order to generalize shape design process, making it independent of origin-destination pair, the domain of homotopy function is set to $[0,1]$ and codomain to $[-1,1]$, with points $(0,0)$ and $(0,1)$ representing start and end point of en-route segment. This also unifies route shape manipulations, like length calculation, trajectory profile extension, etc. for any possible flight. For a given set of homotopies with respect to symmetric reference functions $h^{i}(x)$ and set of parameters $\alpha^{i}$, that controls homotopies, alternative horizontal route shape in en-route segment is computed using the model presented in subsection 3.3.2. The process is visualized in Fig. 3 step 1. Horizontal route shape is decoded in the step 2' (Fig. 3) by scaling, rotation and translation based on real coordinates of the start and end point.

The length of the alternative horizontal route is always larger than the length of nominal direct route. Therefore, direct mapping between the given vertical profile and the new horizontal route is not possible, and the solution is either to re-simulate flight using the aircraft performance model, which is time consuming, 
or to approximate the vertical and speed profiles to match the new route length. In this work the second approach is used, as it is shown in [18] that it is more efficient to extend the vertical profile at the top of descent in the cruise phase of flight. The result is an acceptable approximation of the profile that respects optimal climb and descent gradients as well as speed profile. Step 2"' in Fig. 3 explains a process of trajectory profile extension. Based on a horizontal route extension and initial trajectory state vector $\mathbf{S}$, trajectory profile is extended by adding (multiplying) flight segments as many times as it takes to make the length of new profile and the length of alternative horizontal route shape equal. Vectors $\mathbf{g}_{i . r}, r=\{0 . . m\}$, marked by a red line in vertical profile in the Fig. 3 , are copies of the original vector $\mathbf{g}_{\boldsymbol{i}}$. The final trajectory is computed by matching samples of new profile (vectors $\mathbf{g}_{\boldsymbol{r}}$ ) with the new horizontal position vectors $\mathbf{p}_{r}^{*}, r=\{0 . . n+m\}$ completed with time.
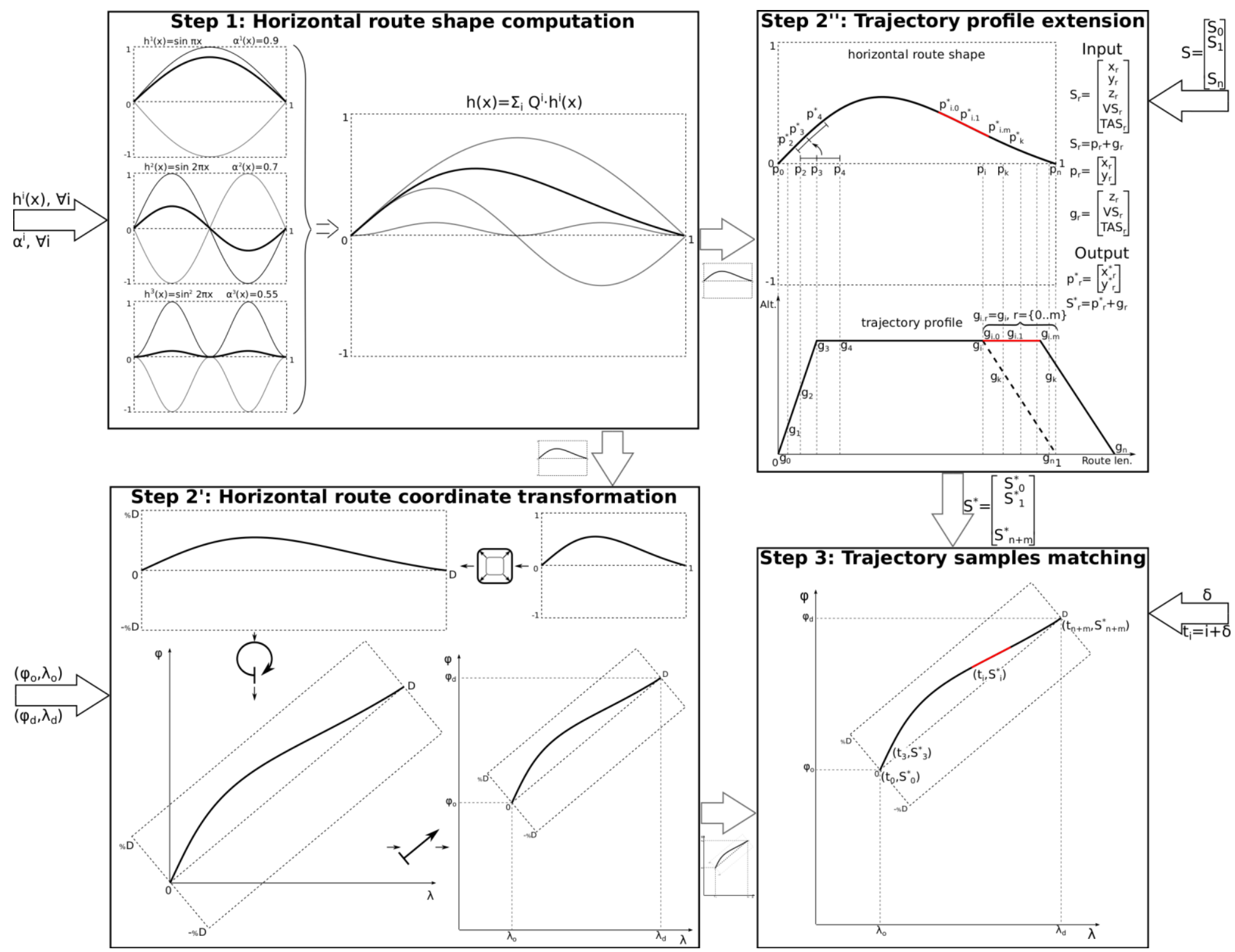

\subsection{RTP mathematical formulation}

\subsubsection{Nomenclature}

F the set of flights $f$;

$\mathrm{A}_{f} \quad$ the set of indices of alternative vertical profiles for flight $f, \mathrm{f} \in \mathrm{F}$;

M the number of symmetrical homotopies;

$a_{f j} \quad$ binary decision variable; equals to 1 if the flight $f$ chooses the alternative vertical profile $j$, and 0 otherwise, $f \in F, j \in A_{f}$;

$\delta_{f} \quad$ decision variable representing (ground) delay of flight $f, \mathrm{f} \in \mathrm{F}$;

$\alpha_{f}^{i} \quad$ decision variable controlling homotopy $i$ for flight $f, \mathrm{i} \in\{1 . . \mathrm{M}\}, \mathrm{f} \in \mathrm{F}$;

Figure 3: Route generation model 
$t_{f}^{n . a} \quad$ nominal arrival time of flight $f, \mathrm{f} \in \mathrm{F}$;

$t_{f}^{a . a} \quad$ actual arrival time of flight $f, \mathrm{f} \in \mathrm{F}$;

$I_{f_{1} f_{2}} \quad$ flight interaction between flights $f_{1}$ and $f_{2}$, $\mathrm{f}_{1} \in \mathrm{F}, \mathrm{f}_{2} \in \mathrm{F} \backslash \mathrm{f}_{1}$;

$C_{f j} \quad$ initial cost of the alternative vertical profile $j$ for flight $f, \mathrm{f} \in \mathrm{F}, \mathrm{j} \in \mathrm{A}_{f}$;

$c_{f}^{d} \quad$ delay unit cost of flight $f, \mathrm{f} \in \mathrm{F}$;

$c^{g} \quad$ fuel price per kilo;

$F B R_{f j} \quad[\mathrm{~kg} / \mathrm{min}]$ fuel burn rate for the flight $f$ using the vertical profile $j, \mathrm{f} \in \mathrm{F}, \mathrm{j} \in \mathrm{A}_{f}$;

$c^{I} \quad$ flight interaction unit cost;

\subsubsection{Decision variables}

The triple $\left(\delta_{f}, \mathrm{a}_{f}, \alpha_{f}\right)$ represents a decision variable associated with each flight $f$, in order to separate them in the 4D space. To separate trajectories in temporal space, a departure delay $\delta_{f} \in \mathbb{N}$ is assigned, while spatial separation is maintained by assignment of $\left(\mathrm{a}_{f}, \alpha_{f}\right)$ for each flight in order to control their 3D routes. Vertical profile $a_{f}$ is selected from the set of alternative vertical profiles, allowing change of cruising altitude. Vector $\alpha_{f}=\left(\alpha_{f}^{1}, \ldots \alpha_{f}^{\mathrm{M}}\right)$ controls the shape of the horizontal route by controlling homotopy $H_{f}$.

\subsubsection{Constraints}

In practice, flow control deals with a time interval divided into a finite number of periods rather than with a continuous time variable. Therefore departure delay can be treated as discrete by dividing the considered maximum allowed delay $\delta_{\max }$ into $\mathrm{k}$ periods of equal length $\Delta \mathrm{t}\left(\mathrm{k}=\delta_{\max } / \Delta \mathrm{t}\right)$. Then, the set of possible ground delays is: $\Delta=\{0, \Delta \mathrm{t}, \ldots,(\mathrm{k}-1) \cdot \Delta \mathrm{t}, \mathrm{k} \cdot \Delta \mathrm{t}\}$. An important feature of the The Homotopy route generation model is that the shape and length of an alternative horizontal route are bounded by the reference functions. The only restriction is that the parameters controlling homotopies have to take a value between 0 and 1 . Finally, the choice of vertical profile is limited to a pre-defined finite set of possible alternative profiles for each flight.

\subsubsection{Objective function}

The RTP is formulated as an assignment of a vertical profile $\mathrm{a}_{f}$, a vector of parameters $\alpha_{f}$ that controls homotopy $H_{f}\left(\alpha_{f}\right)$ and a delay $\delta_{f}$ for each flight $f$, such that the objective function consisting of the total additional costs to network user and the total cost of flight interaction is minimized. The total flight interaction is calculated as the sum of flight interactions between all pairs of flights. Total additional costs to network users, due to deviation from UPTs, are calculated as sum of:

- cost of alternative vertical profile - due to additional fuel burn and possible late arrival at destination. It is an input parameter associated with each alternative profile $C_{f j}$, that equals zero when a nominal vertical profile is used $C_{f j_{0}}=0$,

- cost of delay - due to late arrival at destination point. It is calculated as the difference between actual and nominal arrival time, where the nominal arrival time $t_{f}^{n . a}$ is an input parameter, while actual time $t_{f}^{a . a}$ is computed by the route generation model. Delay unit costs and aircraft categorization are based on a study presented in [33],

- cost of fuel - due to longer alternative horizontal route. It is calculated based on the airborne delay and fuel burn rate $F B R_{f j}$, an input parameter that depends on aircraft type (flight) and cruising altitude (vertical profile). FBR data were extracted from EUROCONTROL's Advanced Emission Model - AEM that are based on BADA (Base of Aircraft Data) datasets.

\subsubsection{Mathematical model}

$$
\begin{aligned}
& \min \sum_{f \in \mathrm{F}} \sum_{j \in \mathrm{A}_{f}} C_{f j} \cdot a_{f j}+\sum_{f \in \mathrm{F}} c_{f}^{d} \cdot\left(t_{f}^{a \cdot a}-t_{f}^{n . a}\right) \\
& +c^{g} \cdot \sum_{f \in \mathrm{F}}\left[\left(t_{f}^{a \cdot a}-t_{f}^{n \cdot a}\right)-\delta_{f}\right] \cdot \sum_{j \in \mathrm{A}_{f}} F B R_{f j} \cdot a_{f j} \\
& +c^{I} \cdot \sum_{f_{1} \in \mathrm{F}} \sum_{f_{2} \in \mathrm{F} \backslash f_{1}} I_{f_{1} f_{2}}
\end{aligned}
$$

subject to constraints:

$$
\begin{aligned}
& \sum_{j \in \mathrm{A}_{f}} a_{f j}=1, a_{f j} \in\{0,1\}, \forall j \in \mathrm{A}_{f}, \forall f \in \mathrm{F} \\
& \delta_{f} \in \Delta, \forall f \in \mathrm{F} \\
& 0 \leq \alpha_{f}^{i} \leq 1, \forall i \in\{1 . . \mathrm{M}\}, \forall f \in \mathrm{F}
\end{aligned}
$$

The first sum in the objective function (5) represents the total initial cost due to the alternative vertical profiles. The second sum is the total delay cost due to late arrivals at destinations. The third sum is the total cost of additional fuel burned due to a longer routes. Finally, the last sum represent total cost of flight interactions.

The constraint (6) ensures that each flight can be only assigned to one vertical profile. The constraint (7) ensures that every flight delay takes a value from a given set of possible ground delays. Finally, the constraint (8) ensures that the parameters controlling homotopies takes a value between 0 and 1 .

\section{RESULTS AND DISCUSSION}

The RTP problem, presented in the previous section, is known to be NP_HARD problem. In addition it has a non-separable state variables and objective function that is evaluated by simulation (black-box). To solve real instances of problems involving high combinatory in huge state space, one must use stochastic optimization techniques. Due to size of memory required for defining a point in the state space, 
Simulated Annealing (SA) as local search metaheuristic has been selected to address this problem based on the fact that it needs less memory than other techniques.

The RTP model is applied to a large scale real-life example to test its capabilities against conventional conflict-free models.

\subsection{Experimental setup}

\subsubsection{Traffic sample}

Traffic data included traffic in French Metropolitan airspace on August 17, 2008. On given date, there were 8,845 flights in the French airspace. To make the problem size manageable, but still realistic three hour period $(9 \mathrm{~h}-12 \mathrm{~h})$ from morning peak is selected. Finally, the traffic sample includes 1,755 flights, of which: $50 \%$ were overflights, about $10 \%$ were domestic flights, and about $40 \%$ were international flights, having either departure or arrival at French airports. Flights were operated by 50 different aircraft types, that furthermore confirms high heterogeneity of demand.

\subsubsection{Traffic data source and format}

System entry time (departure time or time when flight enters airspace) and system entry and exit points (airport or airborne fix) were extracted from real traffic data obtained from the French civil aviation system for flight plan processing, real-time radar data tracking and visualization - CAUTRA. Then, flights were simulated in the ENAC Traffic Simulator (CATS) [8] using direct flight route. Resulting nominal trajectories with system exit times were recorded. As CATS is discrete model, the final flight data were provided as a sequence of route points sampled every 15 seconds. Each point contains the 4D position, velocity and aircraft intention. Two successive points define flight segment as a direct portion of flight.

\subsubsection{Solution space}

Each flight is simulated using five different cruising altitudes: a nominal altitude, two lower and two higher cruising altitudes, maintaining an optimal vertical profile. At the end each flight is assigned with five vertical profiles corresponding to those flight levels.

The shape of the alternative horizontal route is computed using the route generation model based on three reference function, as in Fig. 3 step 1, and three real parameters $\alpha$ having a value between 0 and 1 .

The maximum ground delay is set to 30 minutes.

\subsubsection{Scenarios settings}

In this work two main scenarios are defined and tested. First, the base scenario, aiming to find a robust solution as a balance between interactions and additional flight costs, and second, that aims at finding a conflict-free solution not taking into account solution robustness. The second scenario is designed in order to evaluate robustness of the base scenario.
For both scenarios same interaction, delay and fuel unit costs are used, with difference in $T S^{*}$, that bounds flight interaction. Interaction is bound to 3 minutes in the base scenario while it is set to zero for conflict-free scenario, meaning that it is sufficient that flight are separated in space or in time.

\subsection{Experimental results}

Scenarios were tested on an Intel Pentium DualCore $2.9 \mathrm{GHz}$ PC with $4 \mathrm{~Gb}$ of RAM.

\subsubsection{Base scenario}

When robustness is taken into account, the number of conflicting points of the initial flight plan (nominal trajectories) was 310,814 . It includes interactions of different magnitude, resulting in EUR 92.66 million $^{1}$ of total initial interaction costs.

In the search for the best robust solution, the optimization algorithm was able to reduce the value of the objective function to 0.19 million of EUR. The algorithm intended to find the best possible balance between interaction and operating costs, hence, there were some remaining interactions located near Paris TMA (marked in Fig. 4a). This was, however, a very challenging task. The best solution was obtained in 20 hours of CPU time, while the solution with objective value in the range of $10 \%$ of the objective of the best known solution was found in less than 500 SA steps in 13 hours. For the reference, excluding flight operating costs from the objective, a robust solution was found in one hour on average.

To find a robust solution, as expected, many flights have been modified, i.e. $87 \%$ of all flights. The majority of flights were assigned an alternative horizontal route, while less than $30 \%$ were delayed or assigned to a nonnominal cruising altitude. The main numerical results are presented in Table 1.

Required trajectory modifications result in an increase of operating cost of EUR 108 per flight on average. This is a very promising result considering that no additional disruptions should be experienced due to increased solution robustness.

Table 1: Experimental results

\begin{tabular}{|c|c|c|}
\hline & Conflict-free & Base \\
\hline Modified flights & $\mathbf{4 5 \%}$ & $\mathbf{8 7 \%}$ \\
\hline Horizontal route & $40 \%$ & $85 \%$ \\
\hline Ground delay & $15 \%$ & $30 \%$ \\
\hline Vertical profile & $2 \%$ & $23 \%$ \\
\hline Total route extension & $\mathbf{0 . 2 5 \%}$ & $\mathbf{0 . 8 \%}$ \\
\hline Avg. per extended flight & $0.58 \%$ & \\
\hline Total en-route delay & $250 \mathrm{~min}$ & $781 \mathrm{~min}$ \\
\hline Total ground delay & $\mathbf{3 4 2} \mathbf{~ m i n}$ & $\mathbf{2 7 4 3} \mathbf{~ m i n}$ \\
\hline Maximum ground delay & $22 \mathrm{~min}$ & $27 \mathrm{~min}$ \\
\hline Avg. per delayed flight & $1.4 \mathrm{~min}$ & $5 \mathrm{~min}$ \\
\hline Avg. flight level change & $\mathbf{1 . 1 9}$ level & $\mathbf{1 . 3 4}$ level \\
\hline \multicolumn{2}{|l}{} & \\
\hline Total additional operating costs & $\mathbf{2 9 , 9 8 4} \boldsymbol{€}$ & $\mathbf{1 9 2 , 4 1 7} \boldsymbol{~}$ \\
\hline
\end{tabular}

${ }^{1}$ This is the value of the objective function and it is not related to the real cost of airspace users 


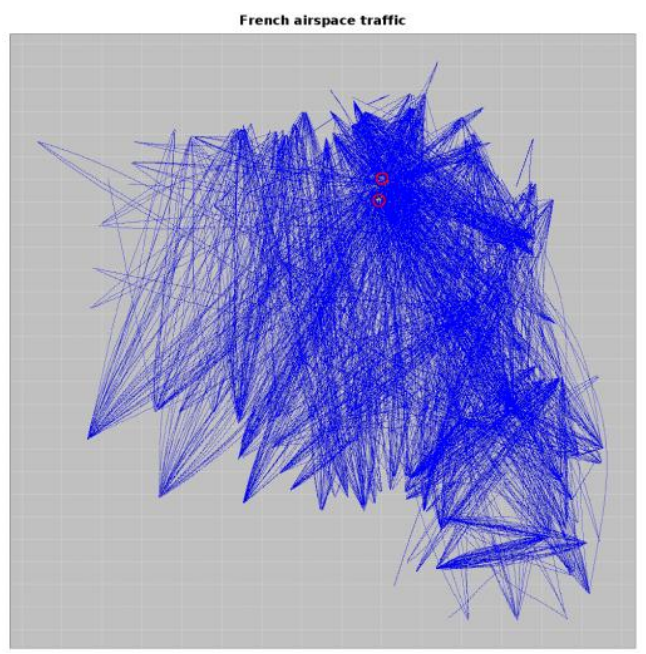

a) Robust trajectories French airspace traffic

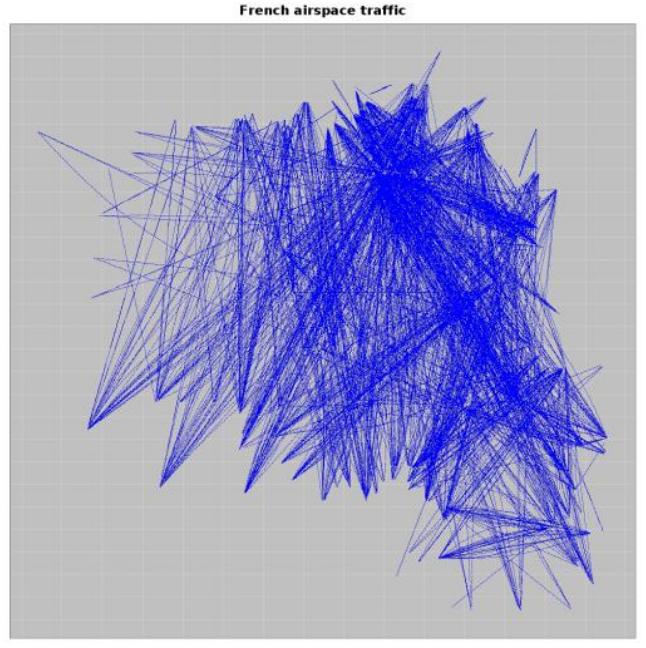

b) Conflict-free trajectories

Figure 4: Resulting solution trajectories

\subsubsection{Conflict-free scenario}

Considering only the conflicts, the general problem is relaxed and a solution to the problem was easier to find. The initial number of conflicting points between nominal flight trajectories was 18,268 , resulting in total initial cost of EUR 18.27 million.

The algorithm was able to find a conflict-free solution (Fig. 4b) and the best found solution had the value of the objective function equal to EUR 29,984. The solution was obtained in 16 hours of CPU time, while the solution with the objective value in the range of $10 \%$ of the objective of the best known solution was found in less than 200 SA steps in 7 hours. To give a reference, if flight operating costs are excluded from the objective function and only conflicts are counted, a conflict-free solution is found in less than 30 seconds.

In the found solution, more than a half of the flights didn't receive trajectory modifications, and once again a horizontal route modification was mostly used to solve potential conflicts. Due to alternative trajectories, operating cost increased EUR 17 per flight on average.
Table 2: Test scenarios settings

\begin{tabular}{|c|c|c|}
\hline Scenario & $\begin{array}{c}\text { Number of } \\
\text { affected flights }\end{array}$ & Delay \\
\hline 1 & 20 & 1.5 minutes \\
\hline 2 & 50 & 1.5 minutes \\
\hline 3 & 100 & 1.5 minutes \\
\hline 4 & 500 & 1.5 minutes \\
\hline 5 (chaos) & 1000 & 1.5 minutes \\
\hline
\end{tabular}

\subsection{Solution robustness testing}

To test solution robustness, conflict-free and robust (base) scenario solutions are imposed to additional flight delays and effects of these disruptions are measured by comparing congestion problems they produce to the system. The magnitude of the congestion problem is measured by the number of newly generated conflicting points. Several scenarios were defined varying in disruption level, as shown in Table 2, depending on the number of affected flights. There were multiple scenario repetitions as the resulting congestion problem is very sensitive to mixture of affected flights.

Distribution of average number of conflicting points over five test scenarios is illustrated in Fig. 5. The graph clearly shows that robust trajectories are less affected by disruptions and therefore cause less congestion problems. It is additionally shown it is easier to solve congestion problems of robust trajectories at the tactical level compared to the one of conflict-free trajectories.

\section{CONCLUSIONS}

This work investigates the potential of robust trajectory planning at strategic, pre-tactical level as a mean to alleviate the en-route congestion in airspace. It introduces a novel approach for route shape generation based on homotopic feature of continuous functions. This approach is capable of generating a large number of routes of different shape with a reasonable number of decision variables.

Application of developed optimization model is illustrated in real-life example. Results show that the model is able to solve real instances of the problem, with computation time that corresponds to the intended use of the model.

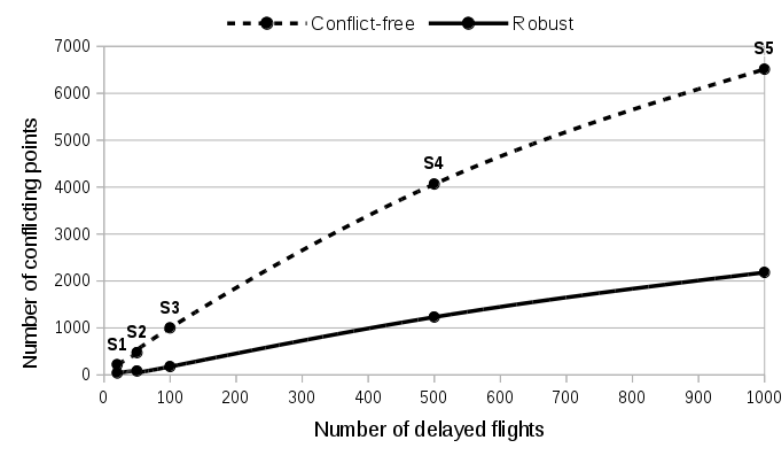

Figure 5: Disruption of conflicting points over test scenarios 
Further, the results indicate that, under certain conditions, solution robustness could be considerably increased at the relative small expense of the solution cost, providing a good alternative to the solutions developed by existing conflict-free trajectory planning models.

Introduction of operating costs into objective significantly increases problem complexity, as due to the rugged shape of the objective function, slow exploration of the search space is inevitable. This influences computation time that should be further improved in order to address larger problem instances. Furthermore, a feature of the homotopy route generation model, that horizontal route is controlled by real value parameters, could be more exploited in future research using field congestion metrics. It is foreseen that in such a case, it would be possible to represent congestion metrics as a function of parameters that controls each homotopy, and not to calculate its value throughout the simulation.

\section{ACKNOWLEDGMENTS}

The research of this work has been supported by research grants of the Ministry of Science and Technological Development, Republic of Serbia, through projects of the Faculty of Transport and Traffic Engineering funded under the 2011-2014 Research programs in technological development: Project TR36033, "A support to sustainable development of the Republic of Serbia's air transport system".

The authors would like to thank Chaimatanan Supatcha whose programming code is used as a basis for the development of optimization model, The French Civil Aviation University (ENAC) for providing traffic data and specially Cyril Allignol for his assistance in CATS data manipulation.

\section{REFERENCES}

[1] Performance Review Commission - PRC, "PRR 2012 - Performance Review Report, An Assessment of Air Traffic Management in Europe during the Calendar Year 2012,"

EUROCONTROL, Brussels, 2013.

[2] IATA Economics, "IATA Economic Briefing Inefficiency in European Airspace," IATA, Montreal, 2013.

[3] STATFOR, the EUROCONTROL Statistics and Forecast Service, "Challenges of Growth 2013 European Air Traffic in 2050," EUROCONTROL, Brussels, 2013.

[4] ICAO, "Global Air Traffic Management
Operational Concept, Doc 9854, First Edition," International Civil Aviation Organization, Montreal, 2005.

[5] NextGen, "NextGen - Implementation Plan," US Department of Transportation, FAA, Washington, 2012.

[6] SESAR consortium, "SESAR Defenition Phase: the Concept of Operations at a glance," EUROCONTROL, Brussels, 2013.

[7] J. Alliot, H. Gruber, G. Joly and M. Schoenauer, "Genetic Algorithms for solving Air Traffic Control conflicts," CENA, Toulouse, 1993.

[8] J. Alliot and N. Durand, "Optimal Reslution of En Route Conflicts," CENA, Toulouse, 1997.

[9] N. Durand, J. Alliot and J. Noailles, "Automatic aircraft conflict resolution using Genetic Algorithms," in Proceedings of the Symposium on Applied Computing (SAC'96), Philadelphia, 1996.

[10] C. Allignol, N. Barnier, N. Durand and J.-M. Alliot, "A new framework for Solving En-Route conflicts," in 10th USA/Europe Air Traffic Management Research and Development Seminar (ATM2013), Chicago, 2013.

[11] L. Blasi, S. Barbato and M. Mattei, "Flight Path Optimization Using Primitive Manoeuvres: A Particle Swarm Approach," Automatic Control in Aerospace, no. 1, 2011.

[12] D. Delahaye, C. Peyronne, M. Mongeau and S. Puechmorel, "Aircraft Conflict Resolution by Genetic Algorithm and B-Spline Approximation," in ENRI International Workshop on ATM/CNS (EIWAC2010), Tokyo, 2010.

[13] K. Zeghal, "A review of different approaches based on force field for airborne conflict resolution," in AIAA - Guidance, Navigation and Control Conference, Boston, 1998.

[14] G. Roussos, G. Chaloulos, K. Kyriakopoulos and J. Lygeros, "Control of multiple non-holonomic air vehicles under wind uncertainty using Model Predictive Control and decentralized navigation functions," in 47th IEEE Conference on Decision 
and Control (CDC'08), Cancun, 2008.

[15] G. Roussos, D. Dimarogonas and K. Kyriakopoulos, "3D Navigation and Collision Avoidance for nonholonomic aircraft-like vehicles," International Journal of Adaptive Control and Signal Processing, vol. 24, no. 10, pp. 900-920, 2010.

[16] J. Kuchar and L. Yang, "A Review of Conflict Detection and Resolution Modeling Methods," IEEE Transactions in intelligent transportation systems, vol. 1, no. 4, pp. 179-189, 2000.

[17] S. Chaimatanan, D. Delahaye and M. Mongeau, "A methodology for Strategic Planning of Aircraft Trajectories using Simulated Annealing," in 1st International Conference on Interdisciplinary Science for Air traffic Management (ISIATM2012), Daytona Beach, 2012.

[18] S. Chaimatanan, D. Delahaye and M. Mongeau, "Strategic deconfliction of aircraft trajectories," in 2nd International Conference on Interdisciplinary Science for Air traffic Management (ISIATM2013), Toulouse, 2013.

[19] N. Dougui, D. Delahaye and M. Mongeau, "A New Method for Generating Optimal Conflict Free 4D Trajectory," in 4th International Conference on Research in Air Transportation (ICRAT2010), Budapest, 2010.

[20] N. Dougui, D. Delahaye, M. Mongeau and S. Puechmorel, "Aircraft Trajectory Planning Under Uncertainty by Light Propagation," Social and Behavioral Sciences, vol. 54, pp. 201-210, 2012.

[21] S. Lan, "Planning for robust airline operation: Optimize aircraft routings and flight departure times to achieve minimum passenger disruptions," Massachusetts Institute of Technology, Massachusetts, 2003.

[22] A. Wieland and C. M. Wallenburg, "Dealing with supply chain risks - Linking risk management practices and strategies to performance," International Journal of Physical Distribution \& Logistics Management, vol. 42, no. 10, pp. 887905, 2012.
[23] M. Snelder, H. van Zuylen and L. Immers, "A framework for robustness analysis of road networks for short term variations in supply," Transportation Research Part A, vol. 46, pp. 828842, 2012.

[24] Episode 3 consortium, "Episode 3 project report: Overall description of the platform and its capabilities," EUROCONTROL, Brussels, 2009.

[25] D. Delahaye and S. Puechmorel, "Air traffic complexity based on Dynamical systems," in 49th IEEE Conference on Decision and Control (CDC2010), Atlanta, 2010.

[26] F. Netjasov, A. Vidosavljevic, V. Tosic, M. Everdij and H. Blom, "Development, validation and application of stochastically and dynamically coloured Petri net model of ACAS operations for safety assessment purposes," Transportation Research Part C, vol. 33, pp. 167-195, 2013.

[27] D. Delahaye, S. Puechmorel, P. Tsiotras and E. Feron, "Mathematical Models for Aircraft Trajectory Design: A Survey," in 3rd ENRI International Workshop on ATM/CNS (EIWAC2013), Tokyo, 2013.

[28] M. Jardin, "Real-Time Conflict-Free Trajectory Optimization," in 5th USA/Europe ATM $2003 R \& D$ Seminar (ATM2003), Budapest, 2003.

[29] D. Delahaye and S. Puechmorel, Modeling and Optimization of Air Traffic, Wiley-ISTE, 2013.

[30] G. P. Collins, "The Shapes of Space," Scientific American, no. 291, pp. 94-103, 2004.

[31] A. Katok and A. Sossinsky, "Introduction to Modern Topology and Geometry," PennState, Pennsylvania , 2006.

[32] A. Vidosavljevic, "Trajectory planning on pretactical and tactical level in Air Traffic Management," UB-FTTE, Belgrade, 2014.

[33] Department of Transport Studies - University of Westminster, "European airline delay cost reference values," University of Westminster for PRU EUROCONTROL, London, 2011. 